\title{
Video Article \\ International Expert Consensus and Recommendations for Neonatal Pneumothorax Ultrasound Diagnosis and Ultrasound-guided Thoracentesis Procedure
}

Jing Liu ${ }^{1,2}$, Dalibor Kurepa ${ }^{3}$, Francesco Feletti ${ }^{4,5}$, Almudena Alonso-Ojembarrena $^{6}$, Jovan Lovrenski ${ }^{7}$, Roberto Copetti ${ }^{8}$, Erich Sorantin ${ }^{9}$, Javier Rodriguez-Fanjul ${ }^{10}$, Karishma Katti ${ }^{3}$, Andrea Aliverti ${ }^{4}$, Huayan Zhang $^{11,12}$, Misun Hwang ${ }^{13}$, Tsu F. Yeh ${ }^{14}$, Cai-Bao Hu ${ }^{15}$, Xing Feng ${ }^{16}$, RuXin Qiu ${ }^{1,2}$, Jing-Han Chi ${ }^{17}$, Li-Li Shang ${ }^{18}$, Guo-Rong Lyu ${ }^{19}$, Shao-Zheng He ${ }^{20}$, Yan-Fen Chai ${ }^{21}$, Zhan-Jun Qiu ${ }^{22}$, Hai-Ying Cao ${ }^{2,23}$, Yue-Qiao Gao ${ }^{1,2}$, Xiao-Ling Ren ${ }^{1,2}$, Guo Guo ${ }^{1,24}$, Li Zhang ${ }^{1,2}$, Ying Liu ${ }^{1,2}$, Wei Fu ${ }^{1,2}$, Zu-Lin Lu ${ }^{1,2}$, Hong-Lei Li ${ }^{1,2}$

${ }^{1}$ Department of Neonatology and NICU, Beijing Chaoyang District Maternal and Child Healthcare Hospital

${ }^{2}$ The National Neonatal Lung Ultrasound Training Base

${ }^{3}$ Division of Neonatal-Perinatal Medicine, Cohen Children's Medical Center

${ }^{4}$ Department of Electronics, Information and Bioengineering, Politecnico di Milano

${ }^{5}$ Dipartimento di Diagnostica per Immagini, Ausl della Romagna, S. Maria delle Croci Hospital

${ }^{6}$ Neonatal Intensive Care Unit, Puerta del Mar University Hospital

${ }^{7}$ Faculty of Medicine, University of Novi Sad, Serbia, Institute for Children and Adolescents Health Care of Vojvodina

${ }^{8}$ Emergency Department, University Hospital of Cattinara

${ }^{9}$ Division of Pediatric Radiology, Department of Radiology, Medical University Graz

${ }^{10}$ Pediatric Intensive Care Unit, Pediatric Service Hospital Joan XXIII Tarragona, University Rovira i Virgil

${ }^{11}$ Center for Newborn Care, Guangzhou Women and Children's Medical Center

${ }^{12}$ Division of Neonatology, Children's Hospital of Philadelphia

${ }^{13}$ Section of Neonatal Imaging, Department of Radiology, Children's Hospital of Philadelphia

${ }^{14}$ Maternal Child Health Research institute, Taipei Medical University and China Medical University

${ }^{15}$ Intensive Care Unit, Zhejiang Hospital

${ }^{16}$ Department of Neonatology, Children's Hospital of Soochow University

${ }^{17}$ Department of Neonatology and NICU, Bayi Children's Hospital Affiliated to the Seventh Medical Center of Chinese PLA General Hospital

${ }^{18}$ Intensive Care Unit, Second Affiliated Hospital of Heilongjiang University of Chinese Medicine

${ }^{19}$ Collaborative Innovation Center for Maternal and Infant Health Service Application Technology, Quanzhou Medical College

${ }^{20}$ Department of Ultrasound, Second Affiliated Hospital of Fujian Medical University

${ }^{21}$ Department of Emergency Medicine, Tianjin Medical University General Hospital

${ }^{22}$ Department of Emergency and Critical Care Medicine, Affiliated Hospital of Traditional Chinese Medicine

${ }^{23}$ Department of Ultrasound, GE Healthcare

${ }^{24}$ The Neonatal Intensive Care Unit, Fifth Medical Center of Chinese PLA General Hospital

Correspondence to: Jing Liu at liujingbj@live.cn

URL: https://www.jove.com/video/60836

DOI: doi:10.3791/60836

Keywords: Medicine, Issue 157, pneumothorax, newborn, infant, lung ultrasound, diagnosis, thoracentesis

Date Published: $3 / 12 / 2020$

Citation: Liu, J., Kurepa, D., Feletti, F., Alonso-Ojembarrena, A., Lovrenski, J., Copetti, R., Sorantin, E., Rodriguez-Fanjul, J., Katti, K., Aliverti, A., Zhang, H., Hwang, M., Yeh, T.F., Hu, C.B., Feng, X., Qiu, R.X., Chi, J.H., Shang, L.L., Lyu, G.R., He, S.Z., Chai, Y.F., Qiu, Z.J., Cao, H.Y., Gao, Y.Q., Ren, X.L., Guo, G., Zhang, L., Liu, Y., Fu, W., Lu, Z.L., Li, H.L. International Expert Consensus and Recommendations for Neonatal Pneumothorax Ultrasound Diagnosis and Ultrasound-guided Thoracentesis Procedure. J. Vis. Exp. (157), e60836, doi:10.3791/60836 (2020).

\section{Abstract}

Pneumothorax (PTX) represents accumulation of the air in the pleural space. A large or tension pneumothorax can collapse the lung and cause hemodynamic compromise, a life-threatening disorder. Traditionally, neonatal pneumothorax diagnosis has been based on clinical images, auscultation, transillumination, and chest X-ray findings. This approach may potentially lead to a delay in both diagnosis and treatment. The use of lung US in diagnosis of PTX together with US-guided thoracentesis results in earlier and more precise management. The recommendations presented in this publication are aimed at improving the application of lung US in guiding neonatal PTX diagnosis and management.

\section{Video Link}

The video component of this article can be found at https://www.jove.com/video/60836/ 


\section{Introduction}

Pneumothorax (PTX) is defined as the presence of air within the pleural space. It is a well-recognized medical emergency condition with high mortality rates, especially in neonates with associated risk factors ${ }^{1,2,3}$. The incidence of PTX is reported to be $1-2 \%$ in term infants and $6 \%$ in premature infants with respiratory distress ${ }^{2,3}$. In addition, lung US (LUS) performed on asymptomatic term infants show that the incidence of mild PTX in these patients can be as high as $10 \%{ }^{2,3}$. Risk factors associated with increased incidence of PTX include meconium aspiration syndrome (MAS), respiratory distress syndrome (RDS), and persistent pulmonary hypertension of the newborn (PPHN) ${ }^{4,5,6,7}$. A 1 min Apgar score $\leq 7$ was associated with a $2.67 x$ increased risk of PTX $(95 \% \mathrm{CI} 1.14-6.25)^{8}$. Increasing peak inspiratory pressure (PIP) during conventional mechanical ventilation has been shown to be a risk factor for PTX, and a PIP increase of $1 \mathrm{~cm} \mathrm{H}_{2} \mathrm{O}$ increases the odds of PTX by $1.46(95 \% \mathrm{Cl} 1.02-2.07)^{8}$. The incidence of PTX in infants with $\mathrm{a}<2,500 \mathrm{~g}$ birth weight (BW) increases almost 10x compared to those with a BW $\geq 2500 \mathrm{~g}^{8}$. Notably, PTX is associated with increased mortality, with an odds ratio of $5.27(95 \% \mathrm{Cl}=1.96-14.17)^{7}$. Apiliogullari et al. reported that aggregate mortality was as high as $30 \%$ in PTX patients while survivors also had an increased rate of bronchopulmonary dysplasia (4.28x vs. controls) ${ }^{9}$. Therefore, early and accurate diagnosis followed by adequate treatment is imperative $e^{3,4,5,6,7,8,9,10,11,12,13,14}$. Lately, less expensive US imaging systems have become readily available, and non-ionizing, fast, and repeatable LUS represents an ideal tool for the diagnosis of neonatal PTX.

PTX is traditionally diagnosed by clinical imaging, auscultation, transillumination, and chest X-ray findings. In some cases of non-tension PTX, watchful waiting is warranted. However, large PTX or tension PTX requires prompt evacuation of the air in the pleural space by thoracentesis. Obtaining a chest X-ray image can be time-consuming and prolong the diagnosis of tension PTX. For these reasons, in many neonatal intensive care units (NICUs), LUS is replacing chest X-rays in diagnosing PTX due to its superior sensitivity and specificity ${ }^{15-17}$. Moreover, LUS has been shown to be more accurate than chest X-rays even for small, non-tension PTX ${ }^{18,19,20,21,22,23,24,25,26,27}$. LUS signs of PTX were first studied and described in adult critical patients. Patients with suspected PTX were scanned with LUS and computed tomography (CT). LUS signs characteristic of PTX were abolition of lung sliding in the B-mode (corresponding to the stratosphere sign in the M-mode), presence of A-lines, and the lung point. In the same study, abolition of lung sliding alone has a sensitivity of $100 \%$ and a specificity of $78 \%$ for PTX. Absent lung sliding together with the presence of A-lines had a sensitivity of $95 \%$ and a specificity of $94 \%$ while lung point alone had sensitivity of $79 \%$ and a specificity of $100 \%^{18}$.

Similarly, the usefulness of LUS to diagnose PTX has been described in infants ${ }^{19,20,21,22,23,24}$. CT could not be used as the benchmark in neonatal patients, thus LUS was compared with chest X-ray and clinical exam findings. Most of the studies included infants with sudden deterioration of their respiratory status, where LUS was performed before or after chest X-ray. The diagnostic accuracy showed a sensitivity of $100 \%$, specificity of $100 \%$, positive predictive value of $100 \%$, and negative predictive value of $100 \% 16,17,18,19$. In cases characterized by large PTX, lung point was absent, which consequently decreased the sensitivity of this sign to $75-95 \%{ }^{21,22}$. The average time to perform the diagnostic tests in these studies was $5.3 \pm 5.6 \mathrm{~min}$ for LUS versus $19 \pm 11.7 \mathrm{~min}$ for a chest X-ray ${ }^{19}$. As expected, LUS showed better diagnostic accuracy than chest transilumination ${ }^{19}$. Keeping in mind that in infants with tension PTX the needle is blindly placed in the second intercostal space at the midclavicular line, it is not surprising to see treatment failure and/or complications ${ }^{6}$. On the other hand, PTX thoracentesis performed under LUS guidance has shown promising results in infants ${ }^{28,29}$.

The neonatal Lung Ultrasound Training Base of China, Chinese College of Critical Ultrasound, as well as the World Interactive Network Focused On Critical Ultrasound China branch have organized this international expert panel that reviewed the latest literature related to neonatal PTX diagnosis and treatment aimed at the improvement in the application of LUS-based diagnosis and treatment of PTX.

\section{Patients and timing of the examination}

The LUS exam can be used on any neonate in respiratory distress. It is indicated in the following situations: 1) Suspicion of PTX in neonates with sudden deterioration of respiratory status; 2) Before and after thoracentesis.

\section{Lung ultrasonography terminology used in PTX diagnosis}

Frequently used ultrasound terms in diagnosis of PTX include: A-line, B-line, confluent B-lines, compact B-lines, alveolar-interstitial syndrome, pleural line, lung sliding, lung pulse, sandy beach sign, and stratosphere sign. The exact definitions of the terms used have been described in detail previously $30,31,32,33,34$.

\section{Protocol}

This work was approved by the Research Ethics Committee of Beijing Chaoyang District Maternal and Child Healthcare Hospital \& Beijing Chaoyang District Bureau of Science, Technology and Information. The study protocol follows the guidelines of the hospital's human research ethics committee.

\section{Ultrasound Exam Preparation}

1. Probe selection

1. Select a high-frequency linear probe $(\geq 10.0 \mathrm{MHz})$ to scan the lungs.

2. Probe disinfection

1. Sterilize the transducer before and after each examination.

3. Preset selection

1. Select the LUS preset.

2. Optimize imaging settings for examination when no LUS preset is available.

1. Select one of the Small Parts presets.

2. Adjust depth to $4-5 \mathrm{~cm}$ using the Depth button. 
3. Adjust the Focus Zone button to have 1 or 2 focuses.

4. Adjust the focus close to the pleural line.

5. Turn on the SRI (Speckle Reduction Imaging) by clicking the button and selecting a level of 2-3 to reduce the speckle noise.

6. Turn on the CRI button (Crossbeam) and select a level of $\mathbf{2}$ to improve the contrast resolution.

7. Select Fundamental Imaging for sharper A-lines or B-lines.

4. Using an ultrasound gel

1. Apply the appropriate volume of warm gel on the transducer to keep it in good contact with the skin surface.

\section{Place the Infant in a Suitable Position}

1. Keep the infant quiet. Use a pacifier when necessary.

2. Keep the infant in a supine, prone, or side position for examination.

\section{Partitioning the Lungs}

1. Six regions: Divide each side of the lung into three regions along the anterior axillary and posterior axillary line. These are the anterior, lateral, and posterior areas. Thus, both lungs are divided into six regions.

2. Twelve regions: Divide each lung into upper and lower lung fields by the nipple connection line. Now there should be 12 regions on both lungs.

\section{Procedure for LUS Imaging}

1. B-mode scanning

1. Press the 2D button or B key to start B-mode scanning.

2. Place the transducer perpendicular to the ribs to start perpendicular scanning. Identify the presence of the pleural line, A-line, and Blines.

3. On real-time US observe whether there is lung sliding or lung point.

4. Rotate the probe $90^{\circ}$ to start parallel scanning.

NOTE: 1) The exam must cover the entire bilateral lung fields. Start at the highest part of the thorax, especially in emergency situations. Because newborns are usually placed in a supine position, this zone is usually located on both sides of the sternum; 2) Bilateral perpendicular scanning is the most important scanning method, while parallel scanning is helpful for diagnosing mild-tomoderate PTX.

2. M-mode scanning

1. Press the $\mathbf{M}$-button to start $\mathbf{M}$-mode scanning. Look for the presence of the stratosphere sign or lung point that indicate PTX. NOTE: Experienced sonographers can detect PTX using only B-mode. M-mode scanning can be used to confirm the B-mode findings if an examiner is less experienced.

\section{Identifying the Presence of PTX}

1. Observe if the pleural line, A-lines, and B-lines exist on B-mode.

2. Observe if lung sliding and lung point exist on real-time US.

3. Observe if the stratosphere sign is present on M-mode.

\section{Identifying the Degree of PTX}

1. Identify the degree of PTX according to the LUS findings.

\section{LUS-guided Thoracentesis}

1. Identify a suitable puncture point.

NOTE: When identifying a suitable puncture point, keep the following in mind: 1) Intercostal space where the pleural line and A-lines exist on B-mode; 2) Intercostal space that presents with a stratosphere sign in M-mode; 3 ) Intercostal space where lung sliding disappears on realtime US.

2. Select an appropriate puncture needle (18-20 G needle or an angiocatheter connected to a $20 \mathrm{~mL}$ syringe and a three-way stopcock).

3. Body positioning

1. Keep the infant in a quiet state. Assure adequate pain control according to the local unit policy.

2. Place the infant in the supine, prone, or side position before thoracentesis, allowing the air on the affected side to rise.

3. Put on a pair of sterile gloves. Disinfect the puncture area.

4. Thoracentesis

1. Keep the infant in a stable position.

2. Evacuate the pleural air by needle aspiration at the selected puncture point. Alternatively, a chest tube may be placed immediately. 
NOTE: In general, thoracentesis achieves good results. Adequate pain control is strongly recommended (a local $1 \%$ lidocaine injection in the dose of $0.5-1.0 \mathrm{mg} / \mathrm{kg}$ or enteral pain control as per unit policy). The use of a pacifier is also encouraged. Larger or tensionPTX is at increased risk of having an underlying bronchopulmonary fistula. It may need a prolonged period of continuous chest tube drainage. Postprocedural LUS evaluation of the affected side is recommended. Cover the insertion site with petroleum gauze once thoracentesis is completed.

\section{Representative Results}

The main purpose of these guidelines is to direct users on how to perform US-guided thoracentesis to treat PTX. Neonatal normal lung appears as a bamboo sign on B-mode US (Figure 1A) and as a seashore sign (Figure 1B) on M-mode US. Lung sliding is clearly evident under real-time US (see Video 1 for lung sliding) ${ }^{31,32,33,34}$

PTX is diagnosed based on the following LUS imaging characteristics: 1) Disappearance of lung sliding. This is the most important sign in the US diagnosis of PTX; 2) Absence of B-lines; 3) Presence of the pleural line and A-lines; 4) On M-mode imaging a normal sandy beach sign is replaced by the stratosphere sign, which is highly specific for PTX; 5) Presence of the lung point in mild to moderate PTX. This sign may not be evident if PTX is large ${ }^{30,31,32,33,34}$. The PTX diagnostic flowchart is presented in Figure $\mathbf{2}^{34}$.

\section{Identifying the degree of PTX}

The severity of PTX can be identified by the several characteristics. 1) Mild PTX: LUS signs of PTX exist in the anterior chest areas only when an infant is in a supine position. The area where lung sliding disappears is approximately $<50 \%$ of the whole lung field or the spared areas exist. Lung point is easily identifiable due to the normally expanded lung. The presence of a spared area generally suggests mild PTX; 2) Moderate PTX: LUS signs of PTX are evident in the anterior and lateral chest areas when the infant is in a supine position. The area where lung sliding disappears is $>50 \%$ of the whole lung field. Identifying the transitional lung point area may be challenging; 3 ) Severe PTX: LUS signs of PTX exist in the anterior, lateral, and posterior lung areas. Lung sliding is absent in all lung areas. There is no identifiable lung point.

\section{Thoracentesis under lung US guidance}

The infant can be placed in the supine, prone, or side position. Slight elevation of the upper body helps obtain more complete air evacuation. If severe PTX is present, the thoracentesis must be performed immediately (Figure 3, Video 2). Place the patient in a prone position (Figure 4A), side position (Figure 4B), or supine position. In the case of tension PTX, continuous air drainage with a chest tube can be used with the infant in a supine position (Figure 4C). In moderate PTX, if thoracentesis is indicated, the site of needle insertion can be anywhere in the field where lung sliding is absent (Figure 5, Video 3). Mild PTX (Figure 6, Figure 7, Video 4, Video 5, Video 6) generally does not require thoracentesis. However, if the primary pulmonary disease of the infant is more severe and the infant presents with clinical deterioration, then thoracentesis may be indicated (Figure 8, Video 7).

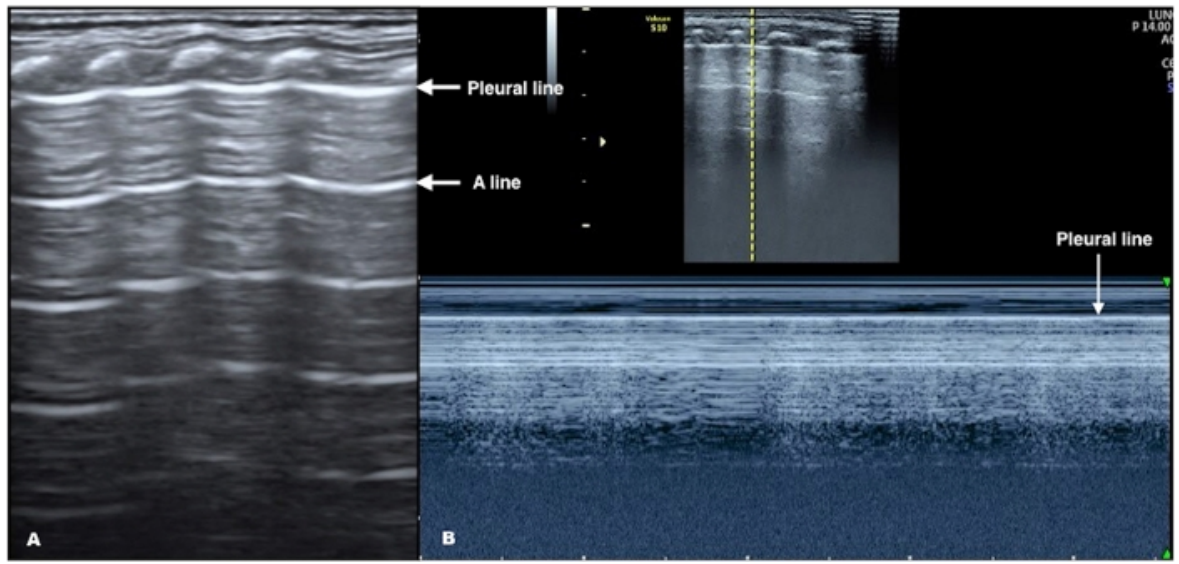

Figure 1: Neonatal Normal LUS. (A) B-mode US: Pleural line and A-lines are smooth, regular, and straight hyperechoic lines, parallel and equidistant from each other. A-lines gradually diminish and finally disappear off the screen. (B) M-mode US: Above the pleural line are linear hyperechoic lines that correspond to the non-moving skin, and subcutaneous and muscle tissue. Below the pleural line is the normal lung tissue that moves with each respiration, leaving a grainy image. These M-mode findings create a seashore sign. Please click here to view a larger version of this figure. 


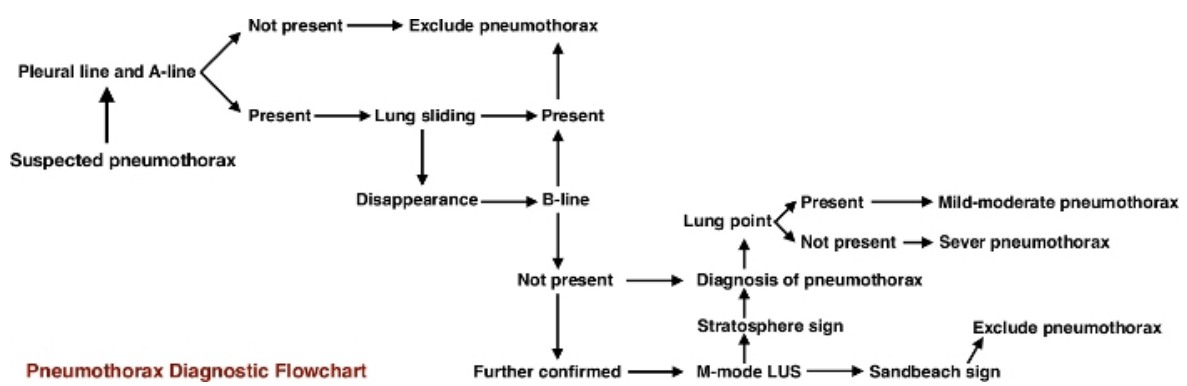

Figure 2: The PTX diagnostic flowchart program. This flowchart program shows that B-mode US is the most important method to diagnose PTX, while M-mode US is helpful to confirm the diagnosis. This figure is reproduced from Liu et al. ${ }^{34}$. Please click here to view a larger version of this figure.

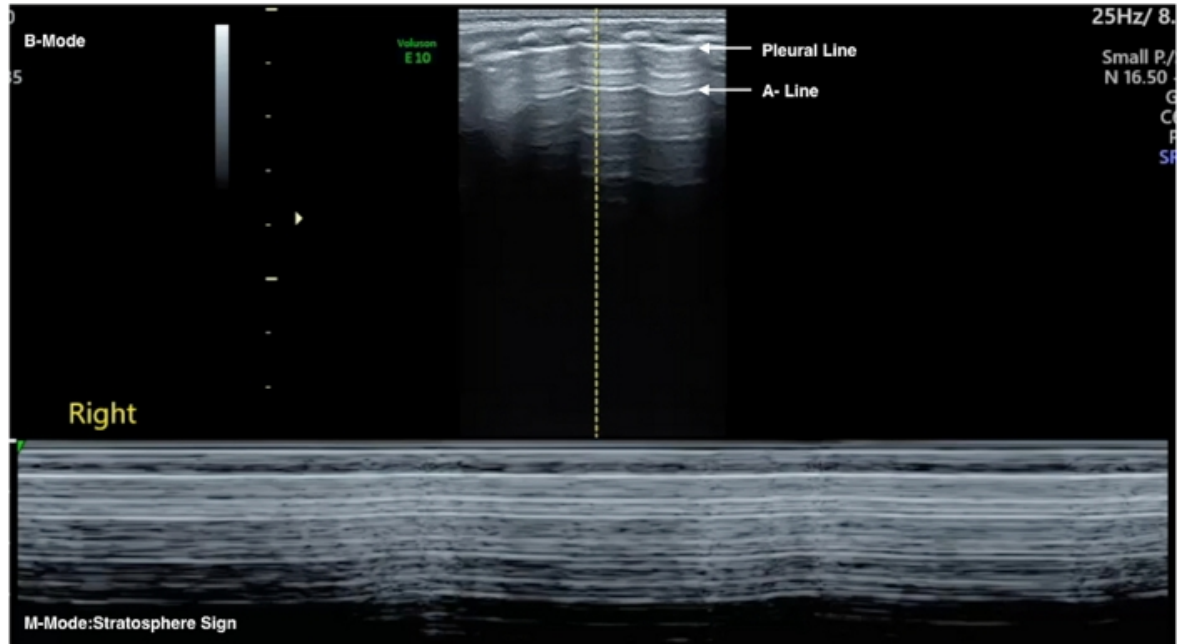

Figure 3: Severe PTX. B-mode US (upper part): The pleural line and the A-lines are present, apparently normal LUS. M-mode US (lower part) shows a stratosphere sign as the lung below the pleural line is displaced by PTX. The absence of lung movement under the pleural line cancels out the normal grainy image. Please click here to view a larger version of this figure.

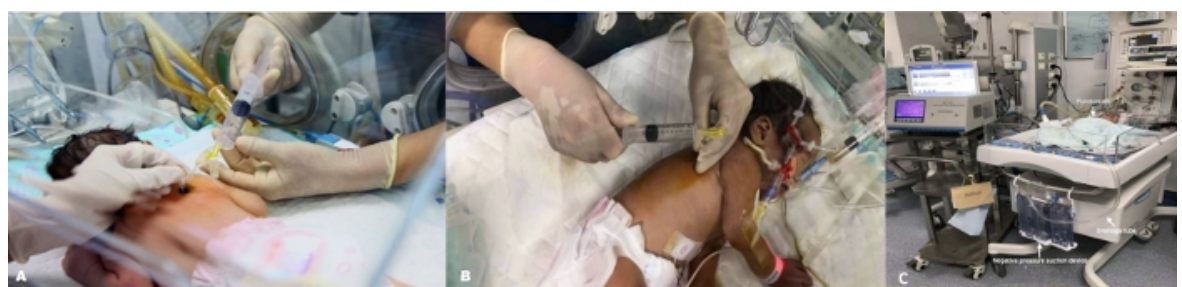

Figure 4: Body position. (A) Infant in prone position. (B) Infant in side position. An angiocatheter is used to evacuate the air from the pleural location. It is connected to a $20 \mathrm{~mL}$ syringe. (C) Chest tube under continuous suction. Please click here to view a larger version of this figure. 


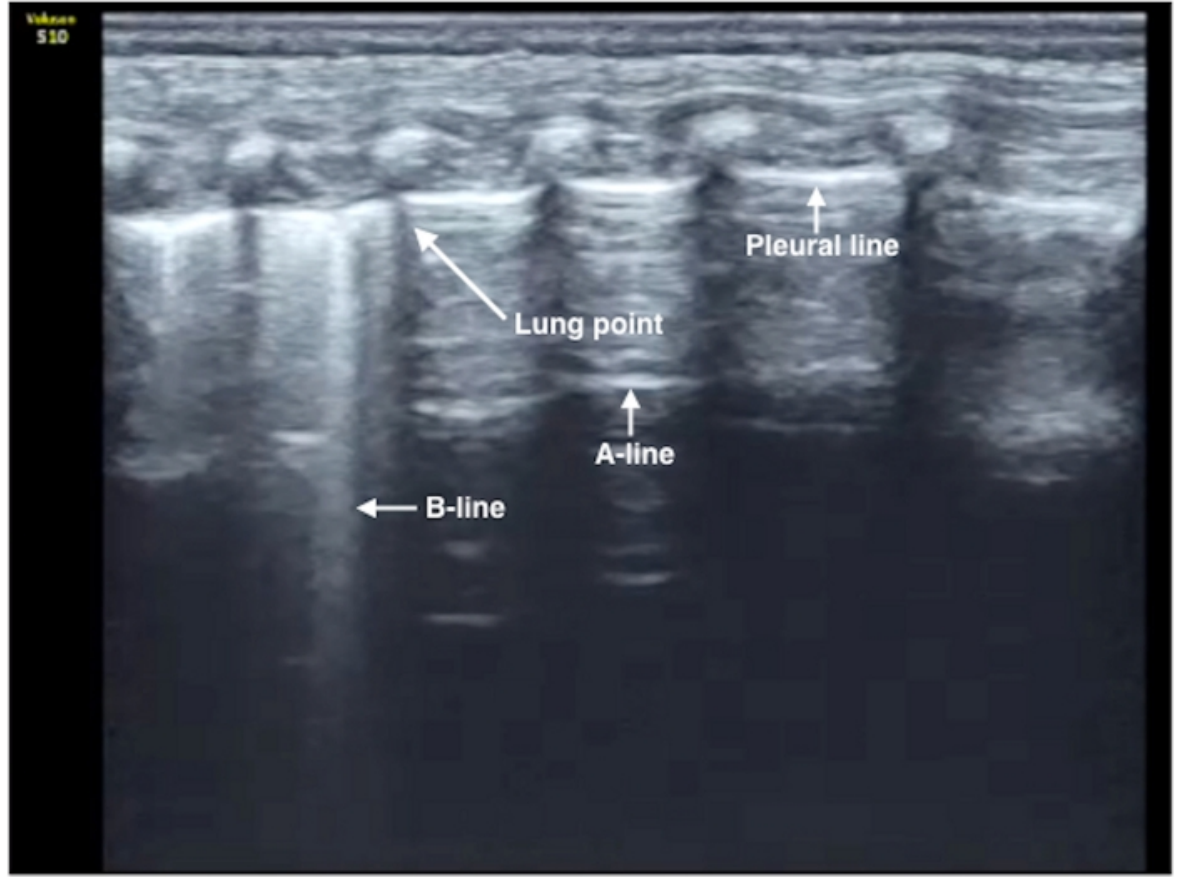

Figure 5: Lung point in moderate PTX. B-mode US: Lung point with an area of disappeared lung sliding that is $>50 \%$ of the whole field, suggesting moderate PTX. Evacuation of air is usually needed with this degree of PTX. The needle puncture site can be selected anywhere in the lung field without lung sliding. Please click here to view a larger version of this figure.

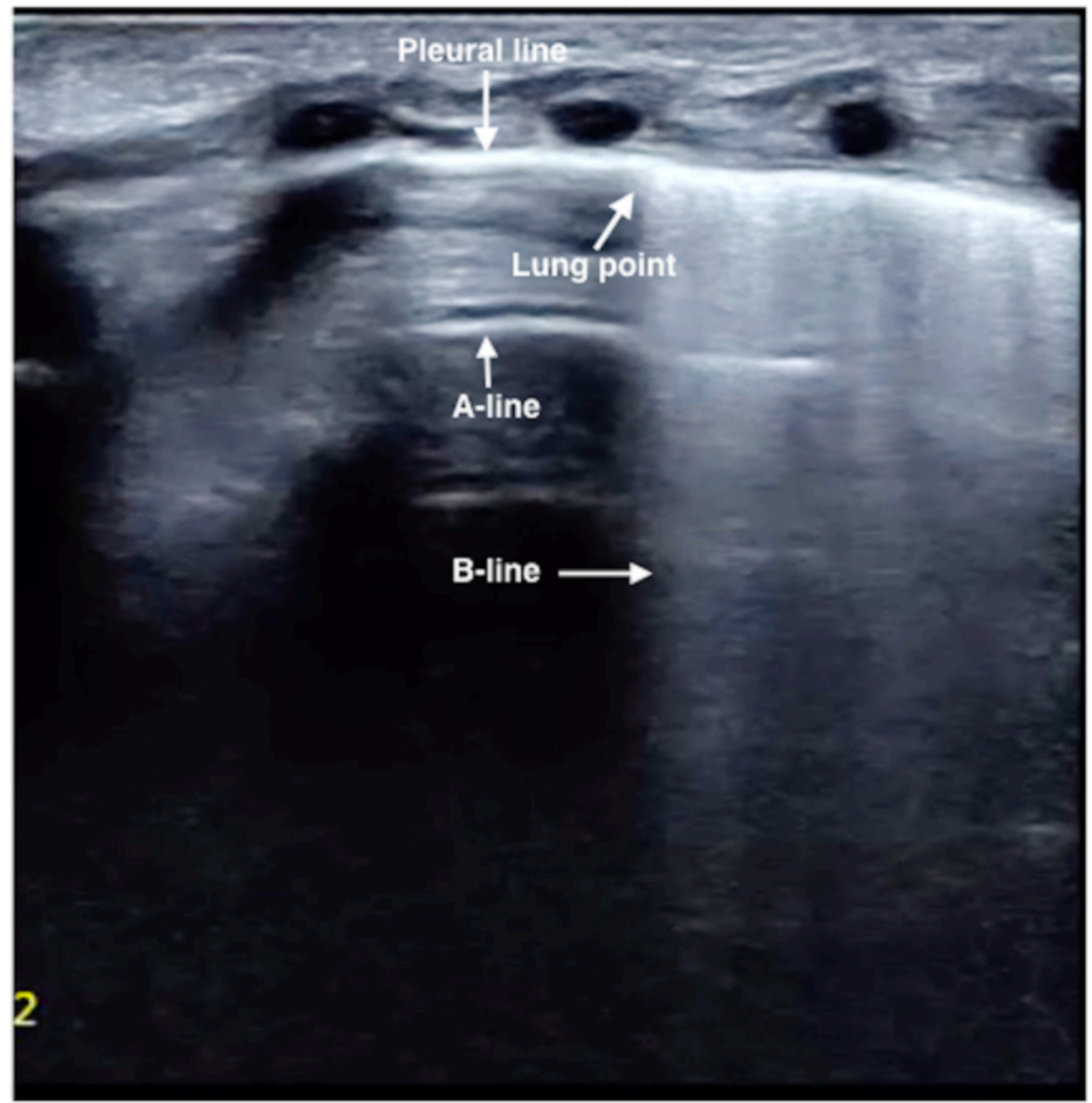

Figure 6: Lung point in mild PTX. B-mode US: Lung point with an area of disappeared lung sliding that is $<50 \%$ of the whole lung field suggests moderate PTX. Evacuation of air is rarely needed with this degree of PTX. Please click here to view a larger version of this figure. 


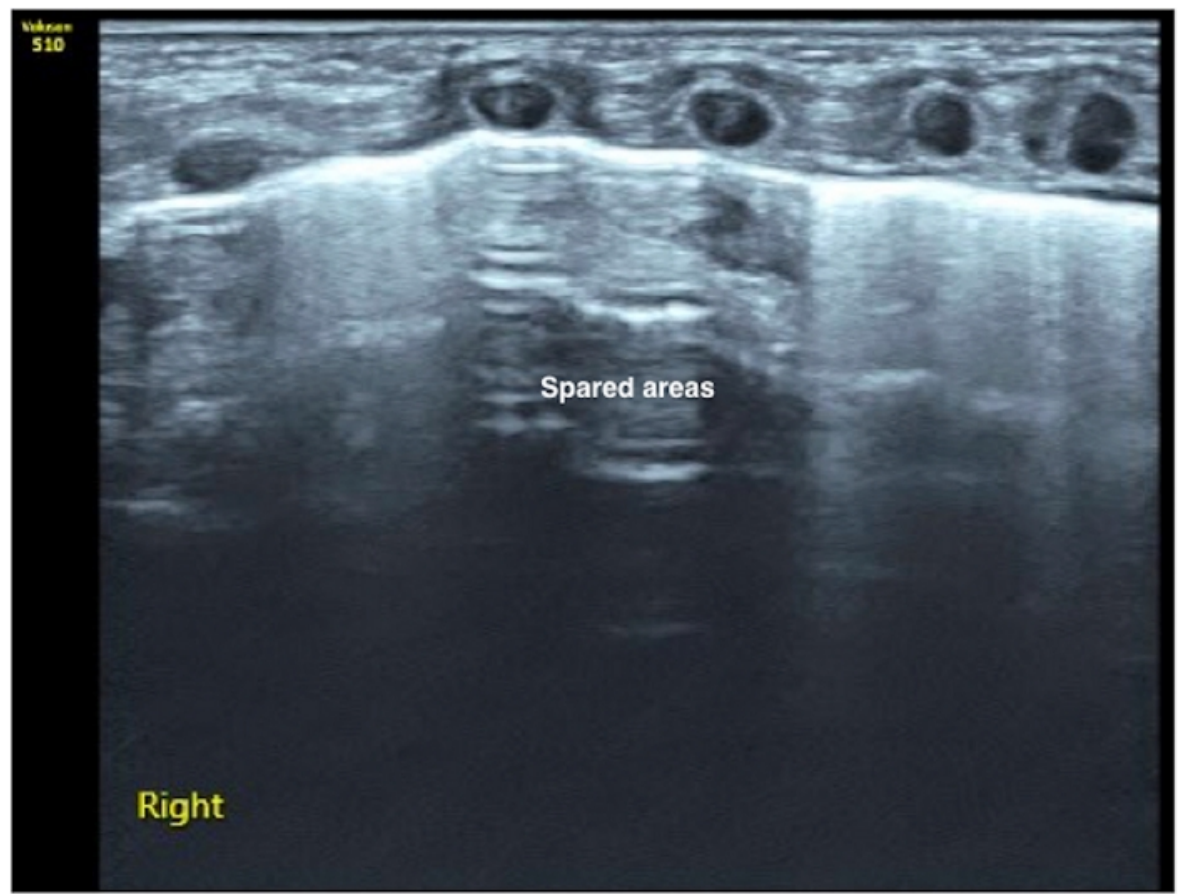

Figure 7: Spared area in mild PTX. The pleural line and A-lines exist in the middle field of the lung while the significant B-lines exist in the upper and lower field of the lung. This kind of lung US sign in known as a spared area. You can find two lung points in this condition. The presence of a spared area generally suggests mild PTX (please also see Video 6). Air evacuation is usually not needed with this degree of PTX. Please click here to view a larger version of this figure.

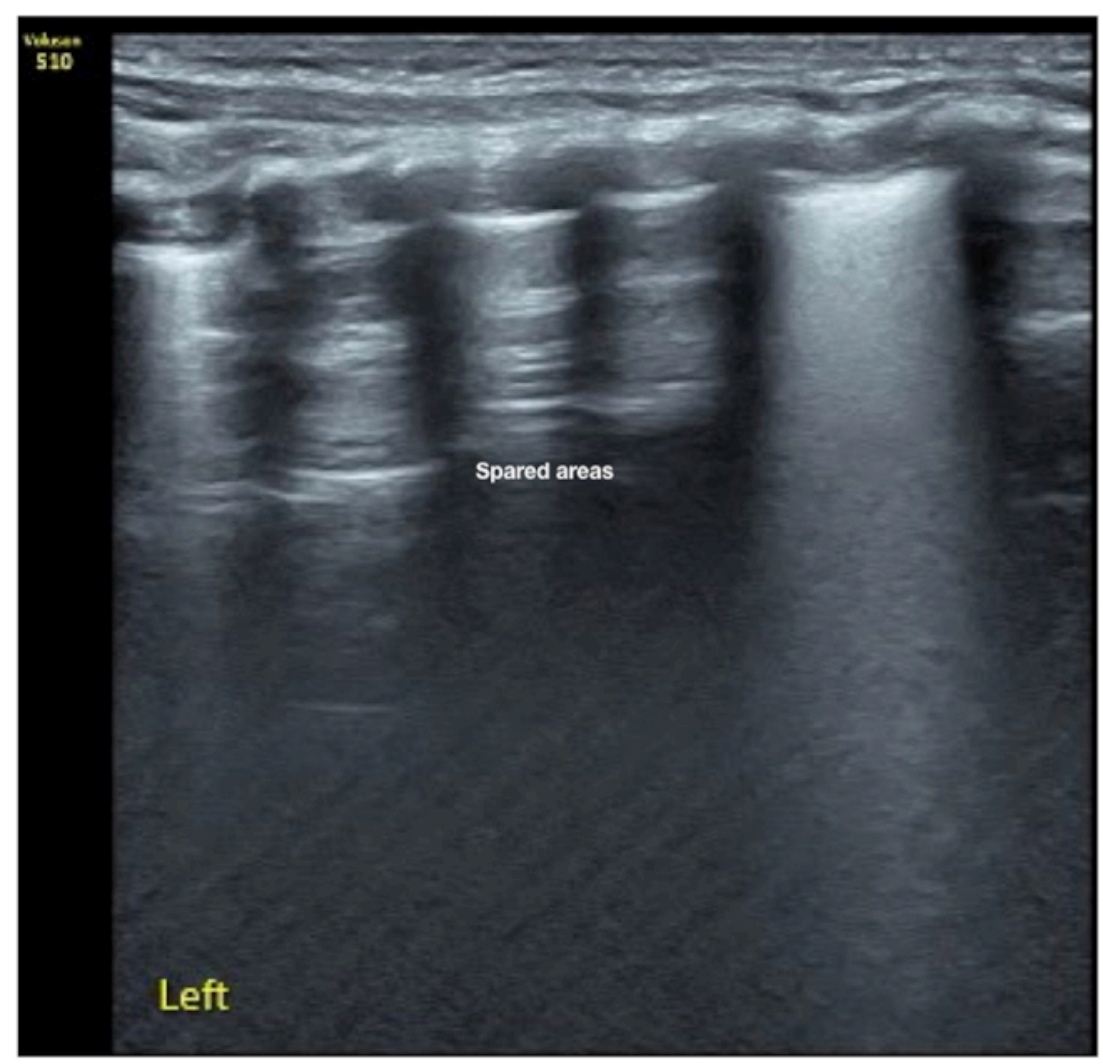

Figure 8: Spared area in mild PTX. A male patient with a gestational age of 41 weeks and birth weight of $3,200 \mathrm{~g}$. The patient was admitted to the NICU because of dyspnea 20 min after birth. LUS showed that spared areas existed in the left anterior chest only. B-mode LUS (Figure 8) and real-time US (Video 7) suggest the presence of mild PTX in the left chest together with pneumonia. Although the infant had only mild PTX, it was accompanied by severe dyspnea not alleviated with mechanical ventilation. Thus, the pleural puncture was performed. The infant's status significantly improved upon drainage of $15 \mathrm{~mL}$ of air from the left chest. Please click here to view a larger version of this figure. 
Video 1: Neonatal Normal LUS. Positive lung sliding under real-time US appears as shimmering of the pleural line. Please click here to download this video.

Video 2: Severe PTX. Absence of lung sliding under real-time US. Please click here to download this video.

Video 3: Lung point in moderate PTX. Under real-time US the lung point presents as an alternate point of lung sliding emergence and disappearance. Please click here to download this video.

Video 4: Lung point in mild PTX. Under real-time US the lung point presents as an alternate point of lung sliding emergence and disappearance. Please click here to download this video.

Video 5: Spared area in mild PTX. Under real-time US, two alternating points of lung sliding emergence and disappearance, indicating two lung points and a spared lung field area. Please click here to download this video.

Video 6: Spared area in mild PTX. A spared area is present in the left anterior chest on real-time LUS. Please click here to download this video.

Video 7: Spared area in mild PTX. On real-time US in the middle field of the lung the lung sliding disappeared, but the pleural line and A-lines are there. In the upper and lower fields of the lung, lung sliding as well as significant B-lines exist. That is the spared area, suggesting mild PTX in the left chest. Please click here to download this video.

\section{Discussion}

LUS for the diagnosis of neonatal PTX is a manageable and timely diagnostic modality $17,19,20,21,22,23,30,35,36,37,38$. Recent animal studies found that the LUS diagnosis of PTX is very accurate and reliable ${ }^{39,40}$. In one of these studies LUS and chest X-rays findings of PTX were compared to CT scans as a point of reference and confirmed that LUS is superior to chest X-rays in diagnosis of small PTX $s^{40}$. In newborn infants with PTX, LUS sensitivity and specificity are also higher than that of chest X-rays ${ }^{17,19-23,37,38}$, and recent meta-analysis further established that the sensitivity of LUS in diagnosing PTX is nearly $50 \%$ higher than the chest X-ray sensitivity ${ }^{41,42}$.

Identification of the degree of PTX is very important for thoracentesis. However, absolutely accurate quantification of PTX volume by LUS is not easy. Finding the lung point effectively distinguishes the normal lung from the lung being separated from the chest wall by the presence of PTX. Similarly, LUS cannot ascertain the depth of air collection. Some studies have shown that semi-quantification of PTX volume is only reliable for small PTX ${ }^{43}$. Therefore, the comprehensive analysis of vital signs, physical exams, and LUS images are essential before making a decision as to whether or not to perform an invasive procedure such as thoracentesis or thoracostomy ${ }^{44,45}$. A study also showed certain variations among pediatric surgeons in the management of spontaneous PTX. The use of CT, timing of operation, and length of observation for air leak before performing surgery have not been adequately standardized ${ }^{44}$. Recent systemic reviews showed no significant difference between thoracentesis and chest tube placement with regards to safety and rates of immediate success. However, thoracentesis is associated with decreased pain and duration of hospital stay compared to chest tube thoracotomy ${ }^{6}$. Traditionally, thoracentesis is performed in the $2^{\text {nd }}$ intercostal space at the midclavicular line or $4-5^{\text {th }}$ intercostal space at the midaxillary line with the needle pointed toward the opposite shoulder with a repeat chest Xray after the procedure. This technique may have several disadvantages. It may delay evacuation of the air because the needle may not always be located right above the PTX, rendering the evacuation incomplete. Evacuation drainage may be prolonged due to incomplete evacuation and the need to change the patient's body position. Also, repeated chest X-ray exposure is always needed. Finally, if the needle is not pointed in the right direction, major blood vessels can be pierced. LUS not only facilitates the needle aspiration by decreasing the risk of complications, but it also offers real-time observation of postprocedural PTX resolution and lung reexpansion ${ }^{46}$. In summary, compared to the traditional thoracentesis procedure there are several benefits of LUS-guided thoracentesis. These include 1) Convenience: There are no limitations to the infant's body position; 2) Accurate and real-time procedure performance: The procedure can be performed immediately after the LUS diagnosis, precisely aimed at the PTX with simultaneous follow-up of lung reexpansion; 3) Decreased risks of complications: LUS can guide the needle just above the rib, avoiding the blood vessels and allowing the operator to visualize the needle as it enters the pleural space; 4) Pain reduction: Shortening the procedural time as well as accurate needle insertion may alleviate the infant's pain ${ }^{47}$.

Critical steps within the protocol are to diagnose PTX and perform thoracentesis proficiently and accurately. The operator must be skilled in neonatal LUS examination as well as in the neonatal thoracentesis technique. Studies have shown that learning essential LUS skills requires short training programs with a relatively small number of supervised scans ranging between $20-80$ LUS exams $^{34,35}$. Several published guidelines should assist in developing and maintaining those skills ${ }^{30,31,32,33,34}$

Limitations to LUS-guided thoracentesis are: 1) Difficulty to accurately quantify the exact PTX volume; 2) Operator-dependent procedure; 3) Less experienced examiners may mistake PTX for diseases that are similar to it, such as bullae and some congenital pulmonary airway malformations ${ }^{48,49}$

For comprehensive neonatal LUS guidelines, including PTX diagnosis, one can also reference previous publications ${ }^{30,31,32,33,34}$. The diagnosis of PTX using LUS is relatively easy when guiding principles are followed. Formal LUS training allows trainees to quickly acquire these skills ${ }^{50}$. Thoracentesis remains a high-risk procedure, particularly in very low birth weight infants. US-guided thoracentesis offers several potential improvements over conventional landmark PTX management. Further, multicenter studies should aim to quantify the extent of this improvement. A detailed description of US-guided thoracentesis allows for a more standardized approach that should guide both clinical practice and research.

Disclosures

The authors have nothing to disclose. 


\section{Acknowledgments}

We acknowledge all the experts and authors that participated in writing and editing the manuscript.

This work was supported by the Social Development Projects, Beijing Chaoyang District Bureau of Science, Technology and Information (CYSF1922 \& CYSF1820) and the Clinical Research Special Fund of Wu Jieping Medical Foundation (320.6750.15072 \& 320.6750.16092).

We acknowledge the Neonatal Lung Ultrasound Training Base of China, Chinese College of Critical Ultrasound, as well as World Interactive Network Focused On Critical Ultrasound China branch for organizing this work.

We acknowledge the all the staff that worked for the Department of Neonatology and the NICU, Beijing Chaoyang District Maternal and Child Healthcare Hospital, especially the nursing staff who assisted this work, particularly during the process of the video recording.

\section{References}

1. Hermansen, C. L., Lorah, K. N. Respiratory distress in the newborn. American Family Physician. 76 (7), 987-994 (2007).

2. Horbar, J. D. et al. Trends in mortality and morbidity for very low birth weight infants,1991-1999. Pediatrics. 110 (1 Pt 1), $143-151$ (2012).

3. Hadzic, D. et al. Risk factors and outcome of neonatal pneumothorax in Tuzla Canton. Materia Socio-Medica. 31 (1), 66-70 (2019).

4. Bhatia, R., Davis, P. G., Doyle, L. W., Wong, C., Morley, C. J. Identification of pneumothorax in very preterm infants. Journal of Pediatrics. 159, 115-120 (2011).

5. Duong, H. H. et al. Pneumothorax in neonates: trends, predictors and outcomes. Journal of Neonatal-Perinatal Medicine. 7 (1), $29-38$ (2014).

6. Bruschettini, M., Romantsik, O., Zappettini, S., O'Donnell, C. P., Calevo, M.G. Needle aspiration versus intercostal tube drainage for pneumothorax in the newborn. Cochrane Database Syst Rew. 2, cd011724 (2019).

7. Jaroensri, S., Kamolvisit, W., Nakwan, N. Risk factor analysis of pneumothorax associated with persistent pulmonary hypertension of the newborn in Thai neonates. Journal of Maternal-Fetal and Neonatal Medicine. 28, 1-6 (2019).

8. Aly, H., Massaro, A., Acun, C., Ozen, M. Pneumothorax in the newborn: clinical presentation, risk factors and outcomes. Journal of MaternalFetal and Neonatal Medicine. 27 (4), 402-406 (2014).

9. Apiliogullari, B., Sunam, G. S., Ceran S., Koc, H. Evaluation of neonatal pneumothorax. Journal of International Medical Research. 39 (6), 2436-2440 (2011).

10. Smith, J., Schumacher, R. E., Donn, S. M., Sarkar S. Clinical course of symptomatic spontaneous pneumothorax in term and late preterm newborns: report from a large cohort. American Journal of Perinatology. 28 (2), 163-168 (2011).

11. Vibede, L., Vibede, E., Bendtsen, M., Pedersen, L., Ebbesen, F. Neonatal pneumothorax: a descriptive regional Danish study. Neonatology. 111 (4), 303-308 (2017).

12. Garcia-Munoz Rodrigo, F. et al. Perinatal risk factors for pneumothorax and morbidity and mortality in very low birth weight infants. Journal of Maternal-Fetal and Neonatal Medicine. 30 (22), 2679-2685 (2017).

13. Al Matary, A. et al. Characteristics of Neonatal Pneumothorax in Saudi Arabia: Three Years' Experience. Oman Medical Journal. 32 (2), 135-139 (2017).

14. MacDuff, A. et al. Management of spontaneous pneumothorax: British Thoracic Society Pleural Disease Guideline 2010. Thorax. 65 (2), 18-31 (2010).

15. Chen, S. W., Fu, W., Liu, J., Wang, Y. Routine application of lung ultrasonography in the neonatal intensive care unit. Medicine. 96 (2), e5826 (2017).

16. Gao, Y. Q., Qiu, R. X., Liu, J., Zhang, L., Geng, S. S. Two years of clinical practice in the diagnosis of pulmonary diseases by ultrasound instead of X-ray in neonatal ward. Chinese Pediatric Emergency Medicine. 26 (8), 588-590 (2019).

17. Liu, J., Lovrenski, J., Hlaing, A. Y., Kurepa, D. Neonatal lung diseases: lung ultrasound or chest X-ray. Journal of Maternal-Fetal and Neonatal Medicine. (2019).

18. Lichtenstein, D. A. et al. Ultrasound diagnosis of occult pneumothorax. Critical Care Medicine. 33 (6), 1231-1238 (2005).

19. Cattarossi, L., Copetti, R., Brusa, G., Pintaldi, S. Lung ultrasound diagnostic accuracy in neonatal pneumothorax. Canadian Respiratory Journal. 2016, 6515069 (2016).

20. Raimondi, F. et al. Lung ultrasound for diagnosing pneumothorax in the critically ill neonate. Journal of Pediatrics. 175, 74-78.e71 (2016).

21. Liu, J. et al. Lung ultrasonography to diagnose pneumothorax of the newborn. American Journal of Emergency Medicine. 35 (9), $1298-1302$ (2017).

22. Deng, B. Y. et al. Use of Neonatal Lung Ultrasound for the Early Detection of Pneumothorax. American Journal of Perinatology. (2019).

23. Raimondi, F., Yousef, N., Migliaro, F., Capasso, L., De Luca, D. Point-of-care lung ultrasound in neonatology: classification into descriptive and functional applications. Pediatric Research.Jul 20 (2019).

24. Thakur, A., Kler, N., Garg, P. Lung Ultrasound for Detection of Pneumothorax in Neonates. Indian Journal of Pediatrics. 86 (12), $1148-1148$ (2019).

25. Maury, É. et al. Diagnostic ultrasound in pneumothorax. Revue des Maladies Respiratoires. 33 (8), $682-691$ (2016).

26. Gardelli, G. et al. Chest ultrasonography in the ICU. Respiratory Care. 57 (5), 773-781 (2012).

27. Feletti, F., Gardelli, G., Mughetti, M. Thoracic ultrasonography in paediatrics: a technique often neglected. Quaderni ACP. 16 (3), $122-125$ (2009).

28. Migliaro, F., Sodano, A., Capasso, L., Raimondi, F. Lung ultrasound-guided emergency pneumothorax needle aspiration in a very preterm infant. BMJ Case Reports. 2014, 1-3 (2014).

29. Liu, J., Xia, R. M., Ren, X. L., Li, J. J. The new application of point-of-care lung ultrasound in guiding or assisting neonatal severe lung disease treatment based on a case series. Journal of Maternal-Fetal and Neonatal Medicine. (2019).

30. Husain, L. F., Hagopian, L., Wayman, D., Baker, W. E., Carmody, K. A. Sonographic diagnosis of pneumothorax. Journal of Emergencies, Trauma, and Shock. 5 (1), 76-81 (2012). 
31. Liu, J. Lung ultrasonography for the diagnosis of neonatal lung disease. Journal of Maternal-Fetal and Neonatal Medicine. 27 (8), $856-861$ (2014).

32. Volpicelli, G. et al. International evidence-based recommendations for point-of-care lung ultrasound. Intensive Care Medicine. 38, 577-591 (2012).

33. Kurepa, D., Zaghloul, N., Watkins, L., Liu, J. STATE-OF-THE-ART Neonatal lung ultrasound exam guidelines. Journal of Perinatology. 38 (2), 11-22 (2018).

34. Liu, J. et al. Protocol and Guidelines for Point-of-Care Lung Ultrasound in Diagnosing Neonatal Pulmonary Diseases based on International Expert Consensus. Journal of Visualized Experiments. 145, e58990 (2019).

35. Bedettil, G. et al. Evaluation of ultrasound lung comets by hand-held echocardiography. Cardiovascular Ultrasound. 4 (34), (2006).

36. Flato, U. A. et al. Use of lung ultrasonography in the detection of pneumothorax among medical students and emergency physicians. Critical Care. 15 (Suppl 2), P46 (2011).

37. Liu, J., Cao, H. Y., Sorantin, E. Pneumothorax of the newborn. In Liu J, Sorantin E, Cao HY. Neonatal Lung Ultrasonography. Dordrecht, Netherlands, Springer Nature. 1, 111-121 (2018).

38. Nagarsheth, K., Kurek, S. Ultrasound detection of pneumothorax compared with chest X-ray and computed tomography scan. The American Journal of Surgery. 77 (4), 480-484 (2011).

39. Blank, D. A. et al. Lung ultrasound accurately detects pneumothorax in a preterm newborn lamb model. Journal of Paediatrics and Child Health. 52 (6), 643-648 (2016).

40. Hwang, T., Yoon, Y., Jung, D., Yeon, S., Lee, H. Usefulness of transthoracic lung ultrasound for the diagnosis of mild pneumothorax. Journal of Veterinary Science. 19 (5), 660-666 (2018).

41. Alrajab, S., Youssef, A. M., Akkus, N. I., Caldito, G. Pleural ultrasonography versus chest radiography for the diagnosis of pneumothorax: review of the literature and meta-analysis. Critical Care. 17, r208 (2013).

42. Alrajhi, K., Woo,M. Y., Vaillancourt, C. Test Characteristics of Ultrasonography for the Detection of Pneumothorax: A Systematic Review and Meta-analysis. Chest. 141 (3), 703-708 (2019).

43. Volpicelli, G. et al. Semi-quantification of pneumothorax volume by lung ultrasound. Intensive Care Medicine. 40 (10), $1460-1467$ (2014).

44. Pocivalnik, M. et al. Pneumothorax during mechanical ventilation-therapeutic options in term and preterm neonates. Klinische Pädiatrie. 225 (7), 389-393 (2013).

45. Williams, K., Baumann, L., Grabowski, J., Lautz, T. B. Current practice in the management of spontaneous pneumothorax in children. Journal of Laparoendoscopic \& Advanced Surgical Techniques. 29 (4), 551-556 (2019).

46. Ng, C., Tsung, J. W. Point-of-care ultrasound for assisting in needle aspiration of spontaneous pneumothorax in the pediatric ED: a case series. American Journal of Emergency Medicine. 32 (5), 488.e3-8 (2014).

47. Liu, J, Chen, X. X., Wang, X. L. Ethical issues in neonatal intensive care units. Journal of Maternal-Fetal and Neonatal Medicine. 29 (14), $2322-2326$ (2016).

48. Karacabey, S. et al. Use of ultrasonography for differentiation between bullae and pneumothorax. Emergency Radiology. 26 (1), 15-19 (2019).

49. Aziz, S. G., Patel, B. B., Rubio, E. R. The Lung Point Sign, not Pathognomonic of a Pneumothorax. Ultrasound Quarterly. 32 (3), $277-279$ (2016).

50. Shumbusho, P. J. et al. Accuracy of resident-performed point-of-care lung ultrasound examinations versus chest radiography in pneumothorax follow-up after tube thoracostomy in Rwanda. Journal of Ultrasound in Medicine. (2019). 